Voix et Images

volxetimages

\title{
Lecture, écriture et intertextualité dans Volkswagen Blues
}

\section{Anne Marie Miraglia}

Volume 15, numéro 1 (43), automne 1989

Jacques Poulin

URI : https://id.erudit.org/iderudit/200815ar

DOI : https://doi.org/10.7202/200815ar

Aller au sommaire du numéro

\section{Éditeur(s)}

Université du Québec à Montréal

\section{ISSN}

0318-9201 (imprimé)

1705-933X (numérique)

Découvrir la revue

\section{Citer cet article}

Miraglia, A. M. (1989). Lecture, écriture et intertextualité dans Volkswagen

Blues. Voix et Images, 15(1), 51-57. https://doi.org/10.7202/200815ar d'utilisation que vous pouvez consulter en ligne.

https://apropos.erudit.org/fr/usagers/politique-dutilisation/ 
DOSSIER 51

\title{
Lecture, écriture et intertextualité dans Volkswagen Blues
}

\author{
par Anne Marie Miraglia, Université de Toronto
}

Que l'écriture se prenne souvent elle-même comme sujet des romans de Jacques Poulin est un phénomène incontestable. Mais nulle part est-ce aussi flagrant que dans son roman Volkswagen Blues ${ }^{1}$. C'est à la fois le récit de la quête d'un frère à travers le continent américain et le récit de lectures devenues écritures. Roman autoreprésentatif mettant en scène un couple assez curieux, un écrivain québécois âgé de 40 ans et une jeune Métisse, personnage avide de lectures, Volkswagen Blues se réfléchit non seulement à travers un personnage écrivain, mais aussi à travers un personnage lecteur que j'appellerai le «lecteur fictif».

Si le texte, dont la focalisation est celle de Jack Waterman, nous répète constamment et de plusieurs façons que ce dernier est un écrivain à la fois en quête de son frère et en quête d'un sujet pour son prochain roman (p. 42-43, 289), il ne néglige pas de nous signaler, en même temps et à plusieurs reprises, que la Grande Sauterelle ou Pitsémine est maniaque des livres (p. 169), qu'elle lit avec voracité (p. 41) et qu'elle emprunte des livres aux bibliothèques et aux librairies (p. 42). Par ailleurs, le Volkswagen lui-même déborde de livres:

Il y avait des livres dans tous les recoins du Volkswagen. À ceux que l'homme avait mis dans ses bagages en partant de Québec s'étaient ajoutés les livres qu'il avait achetés ou que la fille avait «empruntés» en cours de route. Il y en avait dans le compartiment aménagé derrière le siège du conducteur; dans le coffre à gants où dormait le chat; derrière et sous le siège du passager; sur la deuxième tablette de l'armoire [...] Quel que fût l'endroit où l'on se trouvait dans le minibus, on avait toujours un livre à portée de la main. (p. 158)

Dans Volkswagen Blues, lire et écrire sont des actes qui sont représentés en relation d'interdépendance. Ainsi, la moitié du chapitre 4 , intitulé «L'écrivain idéal», thématise la lecture avant d'aborder la question de l'écriture. Ce quatrième chapitre soulève deux différents types de lecture: celle d'un lecteur partial, manquant de discernement, et celle d'un écrivain. L'écrivain Jack Waterman est avant tout un lecteur, lecteur de ses propres créations, bien sûr, mais surtout lecteur des productions d'autrui:

1 Jacques Poulin, Volkswagen Blues, Montréal, Québec/Amérique, 1984, 290 p. (Littérature d'Ámérique). Les références à ce titre, désormais entre parenthèses, renverront toutes à cette édition. 
Tandis que la Grande Sauterelle dévorait tous les livres qui lui tombaient sous la main, Jack Waterman était un lecteur inquiet et parcimonieux. Il avait ses auteurs favoris, dont il avait lu tous les livres [...] Et il avait ses livres préférés, qu' il relisait souvent [...]. (p. 42)

Quand le lecteur fictif se dit écrivain, quand l'écriture est sujet du récit, il est souvent évident qu'une certaine lecture précède et agit sur le romancier fictif. Les romans auxquels il se réferre peuvent influencer directement ou indirectement sa création. L'intertextualité, procédé d'autoreprésentation, attire l'attention sur la production et la réception du texte littéraire. Le lecteur fictif, figure d'autoreprésentation, facilite l'insertion des phénomènes d'intertextualité et met en jeu la problématique de l'interprétation du texte.

Dans Volkswagen Blues, le problème de la lecture se pose dès le début: le texte ancien à l'endos de la carte postale envoyée par Théo à Jack Waterman ${ }^{2}$, quinze ans auparavant, est tout à fait illisible (p. 12). La carte postale, selon Jack, serait un appel au secours, un signal de détresse (p. 78). Pitsémine et Waterman se mettent donc à déchiffrer (p. 15) le texte ancien afin de comprendre le message (p. 15) de Théo. La mère de Pitsémine, femme de ménage au musée de Gaspé, leur facilite la tâche en leur signalant l'existence de deux affiches - la première reproduit un extrait original du voyage de Jacques Cartier tandis que la deuxième en est la réécriture moderne (p. 18). Aussi, Volkswagen Blues illustre-t-il que le décodage d'un texte se fait souvent à l'aide d'autres textes.

L'activité de Jack et de la Grande Sauterelle, l'identification et le déchiffrement qu'ils font de l'extrait du récit de Jacques Cartier, les rapproche du lecteur réel. Ils en sont des analogons: ils lisent, décodent et ensuite interprètent le texte afin de saisir les intentions de Théo (p. 78): Si votre frère s'est donné la peine de faire imprimer un texte ancien sur une carte postale, $j$ 'imagine qu'il avait une idée derrière la tête. C'est une sorte de message qu'il vous envoyait, vous ne pensez pas? (p. 15)

L'ambiguîté caractérisant Théo, la dichotomie héros/bum, est en partie le résultat des interprétations conflictuelles provenant des mêmes données. Il en est ainsi, par exemple, du vol de la vieille carte du père Nicolas Point au Kansas City Museum of History and Science dont fut accusé Théo (p. 139). Là où d'autres verraient la criminalité de Théo, Jack trouve de l'héroïsme: Théo était un nationaliste. Peut-être même un membre du F.L.Q. Il pensait que la carte était un document original et il voulait l'enlever aux Américains pour la ramener au Québec. (p. 146)

Non seulement Volkswagen Blues représente des figures du lecteur devant le problème du décodage, mais il oriente aussi et commente explicitement sa propre réception. Il en résulte presque une auto-lecture du texte ${ }^{3}$. Il en est ainsi

2 Jack Waterman est le nom de plume que Théo donna à son frère lorsqu'ils étaient enfants. Waterman est aussi un nom de plume... au sens propre.

3 Par exemple, les titres des chapitres constituent souvent une sorte de lecture du chapitre lui-même: le chapitre 8, «Un endroit bien tranquille» (titre ironique), le chapitre 17, «Le milieu de l'Amérique», et le chapitre 19, «Mourir avec ses rêves». 
au niveau de l'action aussi bien qu'au niveau de son interprétation. Jack Waterman, par exemple, détermine la réaction du lecteur quand il pressent, chez la Métisse, encore une de ses célèbres sorties contre les Blancs (p. 113) ${ }^{4}$. Il en est de même lorsque Waterman imagine que l'on signale la présence de Théo sur une photo. Le narrateur de clarifier: L' homme que le patron du Trieste avait désigné sur la photo n'était pas le frère de Jack. C'était monsieur Ferlinghetti. Lawrence Ferlinghetti, le poète. (p. 263)

Par ailleurs, l'autolecture n'exclut pas une certaine dose d'autocritique qui prévoit également les réactions du lecteur:

[...] On a traversé les deux tiers de l'Amérique en suivant une piste tellement mince... tellement mince et invraisemblable que si on racontait ça aux gens, personne ne voudrait nous croire. Ils nous prendraient pour des fous. [...]

- Et pour finir, on embarque un vieux bonhomme qui fait du pouce et il nous dit que Théo a toutes les chances d'être en Californie et non pas en Oregon! Il n'a jamais vu votre frère de toute sa vie et pourtant il affirme sans aucune hésitation qu'il est allé en Californie. (p. 237)

Volkswagen Blues communique donc avec son destinataire par le biais de ses lecteurs fictifs, lesquels fournissent un modèle possible pour la réception du texte. Jack Waterman prône une lecture lente, consciente de la composition d'une cuvre (p. 46). Quant à Pitsémine, elle croit fermement en la nécessité de la relecture (p. 258) et, en particulier, en une lecture intertextuelle ou dialogique de l'œuvre:

Il ne faut pas juger les livres un par un. Je veux dire: il ne faut pas les voir comme des choses indépendantes. Un livre n'est jamais complet en lui-même; si on veut le comprendre, il faut le mettre en rapport avec d'autres livres, non seulement avec les livres du même auteur. mais aussi avec des livres écrits par d'autres personnes. (p. 169)

Volkswagen Blues non seulement thématise, mais aussi actualise ce modèle de lecture. Les romans de Jack Waterman sont juxtaposés à ceux d'autres romanciers à trois reprises: d'abord lorsque Jack se rend à la librairie Garneau où il lit la première phrase des demiers romans parus et la première phrase de ses propres romans (p. 36); une deuxième fois lorsque la Grande Sauterelle lit les romans de Waterman et l'Hôtel New Hampshire de John Irving (p. 41); une dernière fois lorsque le narrateur évoque les auteurs favoris de Jack (Ernest Hemingway, Réjean Ducharme, Gabrielle Roy, J.D. Salinger, Boris Vian, Richard Brautigan, etc.) et sa timidité vis-à-vis des productions littéraires d'autrui: En général, il n'éprouvait pas le besoin de lire durant les périodes où il écrivait un roman; au cours de ces périodes, qui se prolongeaient de plus en plus, les livres des autres le rendaient impatient et parfois même jaloux. (p. 42) Ces épisodes posent explicitement les romans de Waterman par rapport à la 
production littéraire d'autres écrivains et les situent implicitement par rapport à la littérature américaine. On verra que c'est à partir de la lecture de textes historiques et de textes romanesques américains que s'écrit Volkswagen Blues.

Le texte, nous dit Julia Kristeva, est une productivité, une permutation de textes, une intertextualité 5 . L'écriture se présente ainsi comme travail, comme production. Les textes engendrent des textes: Tout texte se construit comme mosaïque de citations, tout texte est absorption et transformation d' un autre texte. ${ }^{6}$ L'écriture de Volkswagen Blues ne s'écarte pas de la notion bakhtinienne d'ambivalence telle que décrite par Julia Kristeva: Parlant de «deux voies qui se joignent dans le récit», Bakhtine a en vue l'écriture comme lecture du corpus littéraire antérieur, le texte comme absorption de et réplique à un autre texte. ${ }^{7} \mathrm{La}$ reconnaissance de l'intertexte dans Volkswagen Blues permet au lecteur de tenir compte des phénomènes littéraires inhérents à la composition et à la réception du texte. D'après Michael Riffaterre, le texte a la possibilité de contrôler son propre décodage, sa signifiance, par le moyen de l'intertextualité: l'intertexte, quelle que soit son étendue pour un lecteur donné, a des éléments constants entièrement réglés par des impératifs textuels ${ }^{8}$.

Jack Waterman, rappelons-le, est un romancier québécois non seulement en quête de son frère franco-américain, mais aussi en quête d'un nouveau sujet de roman. Aussi lit-il des textes ayant des rapports avec son voyage aux États-Unis. Volkswagen Blues évoque non seulement des ouvrages historiques pertinents à l'exploration de l'Amérique, mais aussi des romans américains dont le thème principal est la poursuite, non sans épreuves, d'un rêve, d'un mythe à travers un espace inconnu, en quête du bonheur et d'une vie meilleure. Les intertextes romanesques américains, tout comme les intertextes historiques, sont évoqués en des points géographiques stratégiques et partagent avec Volkswagen Blues le thème de la quête, spirituelle ou matérielle, suscité pour la première fois par le récit de voyage de Jacques Cartier imprimé sur la carte postale de Théo.

La découverte de l'Amérique dans l'esprit européen du XVIe siècle représentait la possibilité de bonheur et de réussite matérielle, la possibilité de tout oublier et de recommencer dans un monde nouveau, loin de la corruption qui régnait en Europe. Rien de surprenant, donc, que Waterman trouve la carte postale du récit de Jacques Cartier dans le livre de Walter Chapman, The Golden Dream (p. 14) ${ }^{9}$. C'est également de ce texte que Waterman tire la légende d'Eldorado reproduite dans le chapitre 2 de Volkswagen Blues (p. 29).

5 Julia Kristeva, Sémiôtikè. Recherches pour une sémanalyse, Paris, Seuil, 1969. p. 52. Kristeva élabore sa notion d'intertextualité à partir du concept bakhtinien de dialogisme et d'interaction.

6 Ibid., p. 85.

7 Ibid., p. 88.

8 Michael Riffaterre, «La trace de l'intertexte», la Pensée, n² 215, 1980, p. 5.

9 Voir le chapitre 1, «The Gilded Man of Cundinamarca», de The Golden Dream. Seekers of El Dorado, Indianapolis, Kansas City, New York, The Bobbs-Merrill Company Inc., 1967, p. 3-38. Walter Chapman est le pseudonyme de Robert Silverberg. 
Dans ce roman de Poulin, le voyage à travers l'Amérique est à la fois un voyage touristique, historique et littéraire. Il se déroule dans le temps et dans l'espace aussi grâce aux textes suivants: la Grande Aventure de Jacques Cartier de Joseph-Camille Pouliot (p. 19, 22), la Pénétration du continent américain par les Canadiens français de Benoît Brouillette (p. 44-46), Fragiles Lumières de la terre de Gabrielle Roy (p. 44), Toronto during the French Regime (p. 70), The Oregon Trail Revisited (p. 75, 160, 175, 210, etc.), les Indiens du Canada (p. 84), Explorers of the Mississipi de Timothy Severin (p. 123), l'Histoire du Far-West (p. 144) et finalement Beat Angels (p. 264).

L'allusion à ces textes permet à Jacques Poulin de maintenir présent à l'esprit du lecteur à la fois le moment actuel du voyage effectué par Jack Waterman et la Grande Sauterelle, du Québec aux États-Unis, celui effectué par des pionniers et des aventuriers (Étienne Brûlé, Robert Cavelier de La Salle (p. 112), Lamothe-Cadillac, Henri de Tonti, Nicolas Perrot, Pierre-Charles Le Sueur, Greysolon du Lhut, le père Louis Hennepin, Pierre Pépin, Radisson et Des Groseilliers (p. 113), Louis Jolliet et le père Marquette (p. 117) et celui effectué au XIXe siècle du Québec en Oregon ou en Californie. Quelle que soit son motif, le mouvement se dirige toujours vers l'Ouest. Il est évident que la quête de Théo est simultanément la quête des ancêtres qui avaient quitté l'Europe ou le Québec à la poursuite de la Terre promise, du Grand Rêve de l'Amérique (p. 100). Théo, par exemple, est comparé ou identifié au coureur des bois Étienne Brûlé (p. 77) et, en général, aux pionniers: Théo, comme les pionniers, était absolument convaincu qu'il était capable de faire tout ce qu'il voulait (p. 137, nous soulignons). La quête de Théo et la traversée du continent américain impliquent simultanément l'exploration d'une Amérique jadis française et l'exploration d'une écriture américaine moderne.

Or, il est intéressant de remarquer que Volkswagen Blues met en scène la lecture de romans américains à l'exclusion presque complète d'autres littératures ${ }^{10}$. Si, comme le conçoit Jack Waterman, l'écriture est non pas un moyen d'expression ou de communication, mais plutôt une forme d'exploration (p. 90), il est évident que l'écriture est, dans ce cas, surtout l'exploration de textes américains.

Volkswagen Blues initie la lecture de plusieurs romans américains dont Hotel New Hampshire de John Irving (p. 41), The Valley of the Moon de Jack London (p. 234), On the Road de Jack Kerouac (p. 74, 258) et The Adventures of Augie March de Saul Bellow (p. 108). Comme Volkswagen Blues, trois des intertextes américains pourraient être qualifiés de récits de voyage. The Adventures of Augie March et Hotel New Hampshire ne sont pas spécifiquement des récits de voyage quoique le voyage soit présent. L'insertion des textes romanesques américains est liée, en partie, à la préoccupation de Waterman à l'égard de son frère Théo, une sorte de coureur des bois moderne, à

10 Tous les intertextes romanesques sont américains à l'exception de l'l̂le au trésor du poète et romancier écossais Robert Louis Stevenson. 
son désir de lire des livres pertinents à son voyage aux États-Unis et à son statut de romancier en quête d'une nouvelle matière pour son prochain roman:

Pour l'instant, il n'avait pas de roman en chantier [...] Mais n'écrivant pas, il était en mesure de lire et, comme il pensait sans arrêt à son frère, il cherchait des livres ayant un rapport avec le voyage à Saint Louis. (p. 43)

Le thème dominant dans tous les textes américains évoqués est, comme dans Fragiles Lumières de la terre de Gabrielle Roy, la quête du bonheur, d'un mythe à travers une frontière inconnue. Dans la plupart des cas, il s'agit en particulier de l'Amérique, du rêve américain, des rapports à autrui et de la connaissance de soi. Or, Volkswagen Blues assimile tous ces thèmes inhérents aux intertextes romanesques américains.

Les intertextes américains les plus révélateurs du mythe américain et les plus directement impliqués dans la création et l'interprétation de Volkswagen Blues sont le roman de Jack Kerouac, On the Road, et le guide historique et touristique de Gregory M. Franzwa, The Oregon Trail Revisited (une sorte de réécriture du Oregon Trail, publié en 1847 par le journaliste et pionnier Francis Parkman ${ }^{11}$ ). Ces deux livres clefs sont mentionnés à plusieurs reprises. L'ouvrage de Franzwa sert de guide à Jack Waterman et à la Grande Sauterelle à partir de Kansas City. En fait, le chapitre 19, «Mourir avec ses rêves», n'est que la lecture du livre de Franzwa, l'insertion de la parole d'autrui permettant au lecteur de se familiariser avec le trajet et les épreuves des pionniers du XIX siècle (p. 165) et de s'identifier à ces braves gens qui avaient pris la décision de partir en quête du bonheur et de la fortune dans le wild west américain (p. 179). Ainsi, si les intertextes historiques servent à tracer les premières pérégrinations des explorateurs français jusqu'à Saint Louis, c'est le guide américain qui conduira les protagonistes sur la piste de la conquête de l'Ouest américain.

The Oregon Trail Revisited est l'un des deux livres que les voyageurs découvrent avoir été en possession de Théo lorsque celui-ci fut arrêté à Toronto. Le deuxième est le roman de Jack Kerouac, On the Road. Ces deux récits de voyage, ajoutés à celui de Jacques Cartier à l'endos de la carte postale, sont autant de signes incitant Jack Waterman à quitter le Québec afin d'explorer le continent nord-américain dans une tentative de réappropriation physique et spirituelle. Le roman de Jack Kerouac occupe une place explicite dans Volkswagen Blues à partir de l'arrivée à San Francisco. C'est à San Francisco qu'est née l'écriture spontanée de Jack Kerouac et le mouvement littéraire de la Beat Generation. L'esprit de On the Road, son goût pour l'aventure, le risque, le voyage soutiennent la quête vers Théo, vers cette partie de Jack Waterman qui a oublié de vivre (p. 137). L'élan qui pousse les protagonistes de Kerouac à travers l'Amérique ressemble à celui même qui incite les aventuriers et les pionniers à suivre la Piste de l'Oregon: confiance en soi, désir de liberté, de

11 Gregory M. Franzwa, The Oregon Trail Revisited, Gerald (Montana), The Patrice Press, 1983, p. 67. 
bonheur et d'aventures. Volkswagen Blues absorbe et transforme donc aussi bien The Oregon Trail Revisited que On the Road: le roman de Poulin évoque la lutte historique tracée dans The Oregon Trail Revisited tout en faisant une traversée de l'Amérique qui soit une exploration moderne du passé français (et indien) du continent nord-américain.

La représentation du lecteur dans ce roman de Jacques Poulin est donc motivée. Le lecteur fictif de Jacques Poulin lit des textes ayant des rapports spécifiques avec Volkswagen Blues dont l'écriture est évidemment une écriture-lecture. Le voyage que fait Jack Waterman à travers l'Amérique comprend une quête d'identité basée sur l'appartenance historique, géographique et littéraire du Québécois au continent nord-américain. Aussi, la structure de l'espace textuel de Volkswagen Blues dédouble-t-elle le parcours géographique. Composé de trente-trois brefs chapitres, ce roman nous entraîne de Gaspé, au début de mai, jusqu'à San Francisco, en fin d'été. Dans ce récit de voyage et d'exploration, presque chaque chapitre introduit un lieu différent. La structure du texte réitère ainsi celle du voyage à travers l'Amérique. Le chapitre 17, par exemple, est à la fois le milieu du texte, du voyage et du récit ainsi que le désigne le titre, «Le milieu de l'Amérique»:

Ils étaient partis de Gaspé, où Jacques Cartier avait découvert le Canada, et ils avaient suivi le fleuve Saint-Laurent et les Grands Lacs, et ensuite le vieux Mississipi, le Père des Eaux, jusqu'à Saint Louis, et puis ils avaient emprunté la Piste de l'Oregon et, sur la trace des émigrants du $19 e$ siècle qui avaient formé des caravanes pour se mettre à la recherche du Paradis Perdu avec leurs chariots tirés par des boufs, ils avaient parcouru les grandes plaines, franchi la ligne de partage des eaux et les montagnes Rocheuses, traversé les rivières et le désert encore d' autres montagnes, et voilà qu'ils arrivaient à San Francisco. (p. 255-256)

Le roman prend de cette façon la forme de l'Amérique. Lire Volkswagen Blues, c'est à la fois lire l'exploration d'un espace, d'une conscience, d'une écriture et d'une lecture. 